\title{
Accelerated Parallel Magnetic Resonance Imaging with Multi-Channel Chaotic Compressed Sensing
}

\author{
Tran Duc Tan ${ }^{a}$, Dinh Van Phong ${ }^{a}$, Truong Minh Chinh $^{b}$, and Nguyen Linh-Trung ${ }^{a}$ \\ ${ }^{a}$ University of Engineering and Technology, VNUH \\ ${ }^{b}$ Hue University's College of Education
}

\begin{abstract}
This paper considers to enhance the speed of acquisition in magnetic resonance imaging (MRI). Fast image acquisition offers a number of potential benefits, such as: enhancing image contrast and resolution, avoiding physiological effects or scanning time on patients, overcoming physical constraints inherent within the MRI scanner, or meeting timing requirements when imaging dynamic structures and processes. Often, fast acquisition in MRI is achieved using parallel imaging techniques, such as sensitivity encoding (SENSE). Recently, it has been shown that compressed sensing (CS), which allows reconstruction of sparse or compressible images from a small number of linear measurements, can be used to accelerate the acquisition. In a recent work, we have preliminarily shown that replacing randomness used in $\mathrm{CS}$ with deterministic chaos offers a more practical and efficient single-channel CS framework. In this paper, we extend it to multi-channel chaotic CS in order to provide further gain in the speed of acquisition on top of that offered by existing parallel imaging techniques. Such a gain is obtained by way of reducing the number of samples needed from simultaneously sampling the $k$-space. The performance of the proposed method is analyzed using the point spread function, the transform point spread function, and the reconstructed error.
\end{abstract}

Index terms - fast acquisition, MRI, parallel imaging, SENSE, compressed sensing, deterministic chaos.

\section{InTROdUCTION AND STATE-OF-THE-ARTS}

Magnetic Resonance Imaging (MRI), thanks to the phenomenon of magnetic resonance of tissue nuclei (e.g., the hydrogen nucleus $\mathrm{H}$ ) present in the object under imaging (e.g., the brain), has found various applications in the field of biology, engineering, and material science. In principle, by exciting the object with a time-varying excitation RF pulse, the resonance information of the nuclei can be picked up by a receiving $\mathrm{RF}$ coil. We take the simple case of acquisition of a full two-dimensional (2D) digital image (e.g., a brain slice) of the object to explain how the image acquisition is done. During a series of RF excitations, in which the 2D locations of points on the brain slice are encoded, the receiving coil receives an analog MRI time signal which contains the resonance information at all encoded locations. The encoded locations are often represented in the so-called $k$-space in which the changes of locations during the acquisition time form a smooth trajectory in the $k$-space. A digital MRI signal is then obtained by sampling the time and the $k$-space. The digital MRI image is then obtained (reconstructed) by applying a reconstruction algorithm on the digital signal to obtain the digital MRI image of the brain slice; for example, we apply the 2D Fourier transform on the digital MRI signal from the $k$-space to the pixel domain.

Improvements in speed which MRI signal may be acquired have increased dramatically over recently years. Fast image acquisition in MRI is important in order to enhance image contrast and resolution, to avoid physiological effects or scanning time on patients, to overcome physical constraints inherent within the MRI scanner, or to meet timing requirements when imaging dynamic structures or processes. Parallel MRI (pMRI) is an advanced fast imaging technique to reduce the number of samples using multiple coils to simultaneously collect data. Each coil acquires data corresponding to a portion of the imaging object. There exists some redundancy in the acquired data across all the coils. While the acquisition time is inversely proportional to the number of coils, this redundancy can be exploited to reconstruct the final object image. The reconstruction of the image can be done in the image domain, the $k$-space domain or the $k$ - $t$-space domain.

In the image domain approach, image reconstruction is done by solving a set of linear equations in the image domain. A common technique is SENSE (SENSitivity Encoding) [1] which uses the sensitivity profiles in order to reduce the acquisition time. SENSE-like methods include SPACE-RIP [2] and PILS [3]. SPACE-RIP allows to place arbitrarily RF receiver coils around the object and to use of any combination of $k$-space lines, while PILS utilizes localized sensitivities of each coil and the process to estimate the sensitivity profiles. The selection the $k$-space lines in SPACE-RIP can be made to ensure that the frequencyencoded direction is kept unchanged. This allows us to maintain high signal-to-noise ratio (SNR), minimize artifacts of SENSE-like methods, and reduce considerably the complexity [4].

The $k$-space domain approach uses partial data obtained in all the coils to synthesize the full $k$-space, hence reconstruct the MRI image. In this approach, the SMASH (SiMultaneous Acquisition of Spatial Harmonics) method [5] uses the sensitivity profile of receiver coils as a complementary encoding function. It is limited to suitable combinations of coil arrangement, slice geometry, and the compressed factor. The GRAPPA (GeneRalized Autocalibrating Partially Parallel Acquisitions) method [6] uses spatial encoding with an RF coil, and a robust auto-calibration procedure to improve considerably the reconstruction results and reduce the 
computational complexity when compared to other SMASHlike methods.

Methods in image and $k$-space domain approaches have limitations such as low SNR and aliasing artifacts for high compressed factors. In the $k$ - $t$-space domain approach, the $k$-t SENSE method [7] exploits correlations in both $k$ space and time. It was developed to recover un-acquired $k$ space information in a convenient manner [8]. The UNFOLD (UNaliasing by Fourier-encoding the Overlaps Using the temporal Dimension) method [9] encodes the sensitivity into pre-determined frequency bands. $k$ - $t$ SENSE method can be applied to arbitrary $k$-space trajectories, time-varying coil sensitivities, and various reconstruction problems.

Compressed sensing (CS) shows a significant breakthrough in signal processing when sparse or compressible signals can be recovered from a small set of samples [10], [11]. Exact reconstruction can be achieved by nonlinear reconstruction algorithms such as $l_{1}$ minimization [10] or Orthogonal Matching Pursuit [12]. In the context of signal sampling, CS is seen as random undersampling. This method is important because many signals of interest, including natural images, diagnostic images, videos, speech and music, are sparse in some appropriate domains of signal representation.

Among various applications of $\mathrm{CS}$, it has recently been shown to be successfully applied to MRI for fast acquisition by Lustig, Donoho \& Pauly in [13]. In particular, undersampling is performed in the $k$-space by variable density random measurements. Inspired by this work, further development in the direction of using CS for MRI continues, such as $k$ - $t$ SPARSE [14], CG-SENSE [15], $k$ - $t$ FOCUSS [8], and recently chaotic CS-MRI of the $k$-space [16]. The last method undertakes the deterministic approach in CS, which enjoys several new developments, such as [17], [18], [19], [20].

In a recent work [16], we have preliminarily shown that replacing randomness used in CS with deterministic chaos offers a more practical and efficient single-channel CS framework. In this paper, we extend it to multi-channel chaos-based CS in order to provide further gain in the speed of acquisition on top of that offered by CG-SENSE [13]. The combination of multi-channel chaotic compressed sensing with $k$ - $t$ space approaches such as $k$ - $t$ SENSE or $k$ - $t$ FOCUSS is subject to subsequent work.

The organization of this paper is as follows. Section II describes our proposed multi-channel chaotic compressed sensing for MRI based on two conventional methods: compressed sensing and parallel imaging. Section III presents our simulation results. Conclusions are drawn in the last section.

\section{Proposed MUlTi-CHANNEL CHAOTIC COMPRESSED SENSING FOR MRI}

\section{A. Compressed sensing in MRI}

We consider a discrete signal $\mathbf{x} \in \mathbb{R}^{N}$ and assume that $\mathbf{x}$ is $K$-sparse in the $N$-dimensional space spanned by the set of $N$ basis vectors $\left\{\Psi_{i}\right\}_{i=1}^{N}$, that is:

$$
\mathbf{x}=\sum_{i=1}^{N} \Psi_{i} s_{i}=\Psi_{\mathbf{s}}
$$

where $\boldsymbol{\Psi}=\left[\Psi_{1}, \ldots, \Psi_{N}\right]$ is the sparsifying matrix, and $\mathbf{s}=\left[s_{1}, \ldots, s_{N}\right]^{T}$ is the transform vector, containing exactly $K$ nonzero coefficients, $K \ll N$. When used in MRI, the sparsifying matrix $\boldsymbol{\Psi}$ is an Fourier matrix. Note that when $\mathbf{x}$ is a time-sparse signal, i.e. sparse in the time domain, then $\Psi=\mathbf{I}$. For the simplicity of presentation, we restrict ourselves to sparsity, rather than the more general case of compressibility. In the framework of CS, $\mathbf{x}$ is linearly acquired by an underdetermined system, represented by a measurement matrix $\boldsymbol{\Phi}$. The obtained measurements $\mathbf{y} \in$ $\mathbb{R}^{M}, M<N$, are then given by:

$$
\mathrm{y}=\boldsymbol{\Phi} \mathbf{x}=\boldsymbol{\Phi} \Psi_{\mathrm{s}}=\Theta \mathbf{s}
$$

Given $\mathbf{y}, \boldsymbol{\Phi}$ and $\boldsymbol{\Psi}$, the objective is then to faithfully recover $\mathbf{x}$ (and hence $\mathbf{s}$ ) from $\mathbf{y}$ with the smallest possible value of $M$. If the sparsity information in $\mathbf{x}$ is still fully kept, though being hidden, in $\mathbf{y}$, exact reconstruction of $\mathbf{s}$ is feasible if we find a way to fully restore this sparsity from $\mathbf{y}$. It has been proved that if $\Theta$ satisfies a sufficient condition called the Restricted Isometry Property, then the sparsity information is maintained. A useful indicator for this property is the measure of incoherence. $\boldsymbol{\Phi}$ is incoherent with $\Psi$ in the sense that one cannot sparsify the other [21]. One way to ensure the incoherence is to have $\boldsymbol{\Phi}$ as a random matrix with Gaussian i.i.d. elements. Under such a condition, $\mathbf{s}$ can be faithfully recovered from $\mathbf{y}$ when $M$ is such that $c K \log (N / K)<M<N$, where $c$ is some constant, using various sparse approximation techniques, for examples, $l_{1}$ optimization based Basis Pursuit (BP) [10] or Orthogonal Matching Pursuit (OMP) [12].

In a recent paper [20], we proposed to use a chaotic measurement matrix $\boldsymbol{\Phi}$, which is deterministic, instead of random one. To construct the chaotic measurement matrix $\mathbf{\Phi}$, generate sampled logistic sequence by a deterministic chaotic system, then create the matrix $\boldsymbol{\Phi}$ column by column with this sequence. Elements of the logistic sequence are generated by deterministic chaotic system which is so nonlinear, hence becomes random-like. After that, the reconstruction is also performed by the OMP technique. There, the simulated results indicated that the chaotic approach outperformed the random approach in terms of the probability of exact reconstruction. Moreover, using chaotic CS system also inherits a simpler hardware implementation compared to the random one. To generate a sequence of 'random' numbers, we can use a hardware random number generator (HRNG) or a pseudo-random number generator (PRNG). The HRNG is based on a physical phenomena such as electrical noise from a semiconductor diode or resistor or the decay of a radioactive material. Since the PRNG can generate 'random' numbers by feedback shift registers, it is more practical than HRNG. However, a long register is needed to generate a sequence of numbers that approximates the properties 

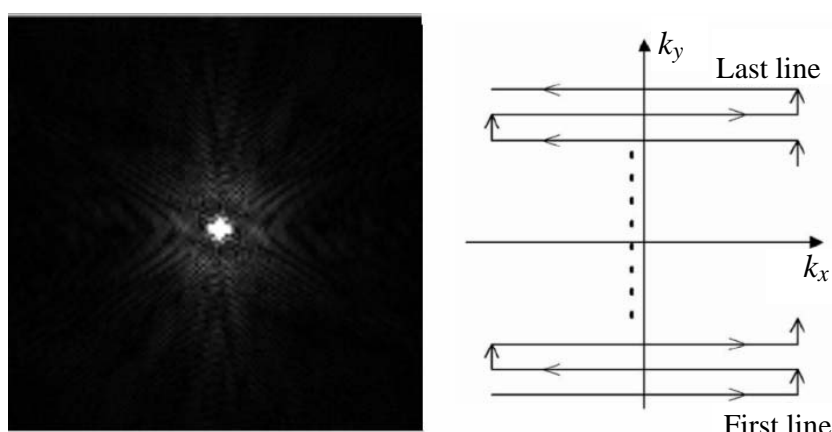

First line

Fig. 1. $k$-space of a brain MR image and a full linear sampling trajectory.

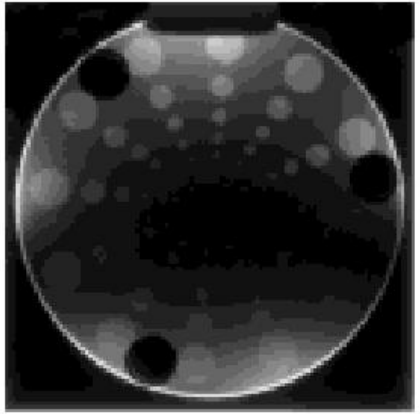

(a)

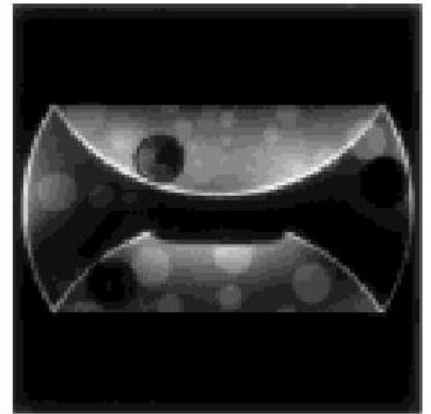

(b)
Fig. 2. Illustration of (a) non-aliasing and (b) aliasing phenomena, using Nyquist sampling and downsamling [1].

of random numbers. Therefore, a large memory and logic circuits are required.

\section{B. Parallel Imaging based on SENSE}

1) Sensitivity encoding (SENSE): The number of excitations, i.e. the number of horizontal lines in the $k$-space trajectory as shown in Fig. 1, determines the total acquisition time. In SENSE, the number of horizontal lines in the trajectory traced by each individual coil is reduced by the number of coils in use. Subsequently, the sensed size of the imaged area is also reduced. The spatial resolution is not changed but aliasing artifacts appear (Fig. 2).

SENSE works in the image domain by removing the aliasing effect caused by combining the individual images, called field-of-view (FOV) images, obtained by individual coils. The inversion of the aliasing transformation for each pixel is calculated individually. Consider the imaging of a slice of the object in the 2D plane $\{x, y\}$. Let $m(x, y)$ be this image. Let $L$ be the number of RF coils. Each coil would have individual values of image intensity. The $k$-space signal obtained from the $l$-th coil is given by:

$$
s_{l}\left(k_{x}, k_{y}\right)=\iint C_{l}(x, y) m(x, y) e^{-j 2 \pi\left(x k_{x}+y k_{y}\right)} d x d y,
$$

where $k_{x}$ and $k_{y}$ encode the information of location along the $x$ and $y$ directions of the image respectively, and $C_{l}(x, y)$ is the sensitivity function of the $l$-th coil. $k=\left\{k_{x}, k_{y}\right\}$ lies in the $k$-space.
Equation (3) shows that $s_{l}\left(k_{x}, k_{y}\right)$ is the Fourier transform of the sensitivity-weighted images $C_{l}(x, y) m(x, y)$. The image acquired by each individual coil, $m_{l}(x, y)$, can then be expressed as the ideal image modulated by the corresponding sensitivity function, by:

$$
m_{l}(x, y)=C_{l}(x, y) m(x, y)
$$

Subsequently, each pixel of the full FOV image can be estimated as:

$$
\hat{m}(x, y)=\mathbf{C}^{H}(x, y) \mathbf{C}^{-1}(x, y) \mathbf{C}^{H}(x, y) m(x, y),
$$

where $\mathbf{C}=\left[C_{1}, \ldots, C_{L}\right]$. In practice, a calibration procedure with the reference images is used to measure the sensitivity of each coil. These reference images must not contain aliasing artifact and noise. Smoothing and extrapolation of the coil sensitivity can be done to obtain an acceptable sensitivity map.

To integrate compressed sensing into SENSE, we consider the k-space full-sampling by discretizing (3) as follows:

$s_{l}\left(k_{x}, k_{y}\right)=\sum_{n_{x}=0}^{N_{x}-1} \sum_{n_{y}=0}^{N_{y}-1} C_{l}\left(n_{x}, n_{y}\right) m\left(n_{x}, n_{y}\right) e^{-j 2 \pi\left(n_{x} k_{x}+n_{y} k_{y}\right)}$

where $N_{x}$ and $N_{y}$ are the numbers of pixels along $\mathrm{x}$ and $\mathrm{y}$ axes of the image. It is obvious that $s_{l}\left(k_{x}, k_{y}\right)$ is viewed as the vector $x$ within the compressed sensing setting. Consequently, we acquire a undersampled signal $\widehat{s}_{l}\left(k_{x}, k_{y}\right)$ in the $l$-th channel by applying the chaotic measurement matrix $\boldsymbol{\Phi}$ to $s_{l}\left(k_{x}, k_{y}\right)$. Since, $\widehat{s}_{l}\left(k_{x}, k_{y}\right)$ is viewed as the vector $y$ within the compressed sensing setting.

2) Conjugate Gradient SENSE (CG-SENSE): As by its original version, SENSE can work only when the $k$-space trajectory is Cartesian, as shown in Fig. 1, rather than other kinds of trajectories. An effective iterative method that can overcome this problem is the CG-SENSE. This method also requires the information of the sensitivity map.

The number of $k$-space samples obtained by undersampling is much smaller than that by full-sampling. MRI reconstruction from the $k$-space samples is performed by Nonlinear Conjugate Gradient (NCG) [13]. The Tikhonov regularization can be given by:

$$
\begin{array}{r}
\arg \min _{m}\left\{\left\|F_{u} \mathbf{m}-\mathbf{y}\right\|_{2}^{2}+\lambda\|\mathbf{m}\|_{2}\right\} \\
\text { subject to } \quad\left\|F_{u} \mathbf{m}-\mathbf{y}\right\|_{2}<\epsilon
\end{array}
$$

where $\mathbf{m}$ is the image vector, $\mathbf{y}$ is the $k$-space measurement vector, $F_{u}$ is the undersampled Fourier operator associated with the measurements, and $\lambda$ is a data consistency tuning constant.

\section{Multichannel compressed sensing using CG-SENSE}

The chaotic measurement matrix is formed in MRI acquisition procedure. We generate the values of $k_{x}$ and $k_{y}$ by a logistic map process, and a couple of $k_{x}$ and $k_{y}$ will determine a coordinate in the $k$-space that will be acquired. However, the distribution of information in $k$-space concentrates nearby the origin and decays when $k_{x}$ and $k_{y}$ increase. 
Fig. 1 shows that most encoded information is concentrated at the origin. Therefore, we convert the distribution of logistic map sequence to Gaussian distribution. The reconstruction is obtained by solving the constrained optimization problem:

$$
\begin{array}{r}
\arg \min _{m}\left\{\left\|F_{u} \mathbf{m}-\mathbf{y}\right\|_{2}^{2}+\lambda\|\mathbf{\Psi} \mathbf{m}\|_{1}\right\} \\
\text { subject to } \quad\left\|F_{u} \mathbf{m}-\mathbf{y}\right\|_{2}<\epsilon
\end{array}
$$

Once the MRI data has been acquired, the reconstruction is performed by the NCG algorithm. Our scheme can be summarized in Algorithm 1.

\section{$\overline{\text { Algorithm } 1 \text { Multi-channel Chaos-based CS for MRI }}$ acquisition}

Step 1: Generate $k_{x}, k_{y}$ that are Gaussian chaotic sequences. The number of $k_{x}, k_{y}$ based on pre-defined compression ratio $r=M / N$.

Step 2: For each channel, determine coordinates in $k$-space based on $k_{x}, k_{y}$ and store as a mask.

Step 3: For each channel, acquire digital data in $k$-space based on the mask and store them in a vector $\mathbf{y}$.

Step 4: Estimate sensitivity maps using polynomial fitting.

Step 5: Perform SENSE reconstruction using conjugated gradient method.

\section{Results AND PERformAnCE}

In the simulation, the data source in use, obtained from [22], is human MPRAGE data from 8-channel head array coil. The data was acquired with the following parameters: $\mathrm{TE}=3.45 \mathrm{~ms}, \mathrm{TR}=2350 \mathrm{~ms}, \mathrm{TI}=1100 \mathrm{~ms}, \mathrm{Flip}$ angle $=7$ deg., slice $=1$, matrix $=128 \times 128$, slice thickness $=1.33 \mathrm{~mm}, \mathrm{FOV}=256 \mathrm{~mm}$.

To compare the efficiency of the design of chaotic measurements, we acquire the data for a series of compression ratios by measurements which are both chaotic and random. Then, we analyze the performance of these systems using the point spread function, the transform point spread function, and the reconstructed error.

To measure the degree of incoherence of a sparsity system, [13] proposed to use the point spread function (PSF), defined as below:

$$
\operatorname{PSF}(i, j)=e_{j}^{*} F_{u}^{*} F_{u} e_{j},
$$

where $e_{i}$ is the $i$-th natural basis vector (having a value of 1 at the $i$ th location and zeros elsewhere). If the $k$-space is fully sampled, then $\left.\operatorname{PSF}(i, j)\right|_{i \neq j}=0$ in the image domain, meaning the system is incoherent.

Fig. 3 shows the PSFs which correspond to $k$-space full-sampling, chaotic undersampling and random undersampling, respectively, with low and high compression ratios. With a low compression ratio, $r=0.05$, the interference between pixels is evident in the both cases of chaotic and random measurements. With a high compression ratio, $r=$ 0.1 , the incoherence at the both cases of chaotic and random measurements is small enough. Consequently, the acquisition produced good reconstructed images. It also can be seen that chaotic $k$-space undersampling and random $k$-space undersampling have similar degree of incoherence.
If the incoherence is analyzed in the transform domain of sparsity, such as the wavelet domain relevant to MRI images, the transform point spread function (TPSF) is used [13], as given by the following equation:

$$
\operatorname{TPSF}(i, j)=e_{j}^{*} \boldsymbol{\Psi} F_{u}^{*} F_{u} \boldsymbol{\Psi}^{*} e_{j}
$$

It can be used to measure how a single transform coefficient affect to other transform coefficients of the measured object. For incoherence, we want to have $\left.\operatorname{TPSF}(i, j)\right|_{i \neq j}$ as small as possible.

Fig. 4 shows the wavelet TPSFs which correspond to the $k$-space full-sampling, chaotic undersampling and random undersampling, with low and high compression ratios. The sparsifying transform here is the 1-level Daubechies-1 wavelet transform. With a low compression ratio, $r=0.05$, our analysis indicated that the interference is spread in all subbands. Whereas, the interference is quite small when a high compression ratio, $r=0.1$, was applied.

Fig. 5 shows the reconstructed images for the $k$-space full-sampling, in comparison with chaotic and random undersampling for several different compression ratios. We can see that the image reconstructed from chaotic measurements is equivalent to random ones. Then, we determine, for each compression ratio, the error in the reconstructed image as compared to the original image. Suppose that $I$ is an $N \times M$ original image and $\hat{I}$ is the reconstructed image. We define the error between them by:

$$
e=\frac{1}{N \times M} \sum_{i=1}^{N} \sum_{j=1}^{M}\left|I_{i j}-\hat{I}_{i j}\right| .
$$

Fig. 6 shows the results of this comparison. We can see that, for compression ratios that are larger than 0.1 , the image reconstructed from chaotic measurements has smaller average error than the image reconstructed from random measurements. Our results confirm the success of replacing random measurements by chaotic measurements.

\section{CONCLUSION}

We have successfully combined chaos-based compressed sensing and the CG-SENSE technique in order to accelerate the speed of acquisition in parallel MRI imaging, hence improve the scanning time as well as reduce the hardware complexity by the deterministic approach, while ensuring the quality of reconstructed image. Both of these methods can exploit the information of the coil sensitivity map and the sparsity of the image. The simulation on the chosen MRI image shows that the system is potential for a practical implementation.

Subsequent work could further combine multi-channel chaotic compressed sensing $k$ - $t$ space approach such as $k$ - $t$ SENSE in order to improve the quality of the reconstructed image.

\section{ACKNOWLEDGEMENT}

This work is supported by the QG-10.40 project of Vietnam National University, Hanoi. 


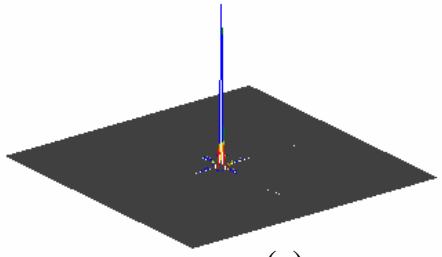

(a)

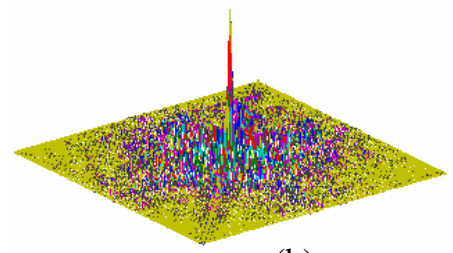

(b)

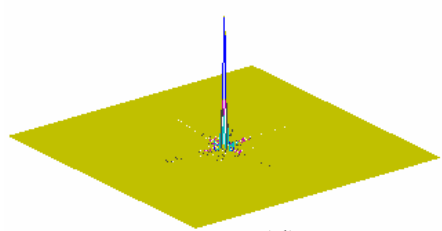

(d)

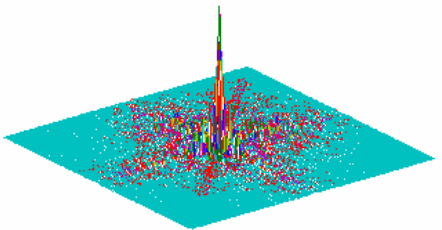

(c)

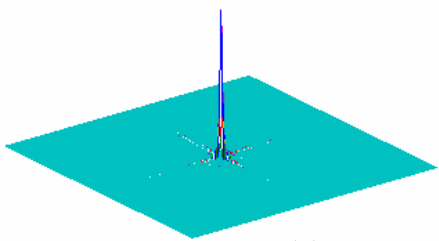

(e)

Fig. 3. PSFs in the image domain for full-sampling (a), chaotic undersampling (b) and random undersampling (c) with the compression ratio $r=0.05$ (b), chaotic undersampling (d) and random undersampling (e) with $r=0.1$

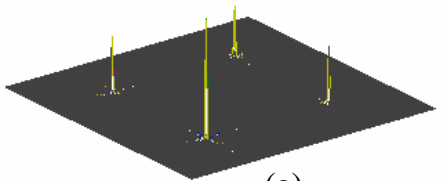

(a)

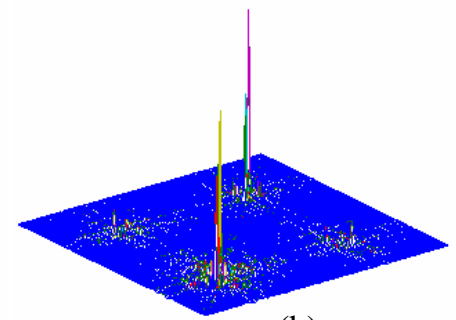

(b)

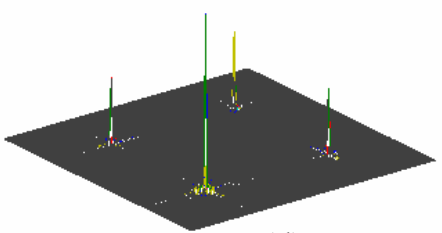

(d)

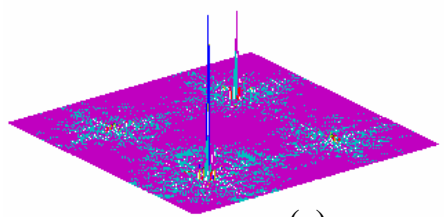

(c)

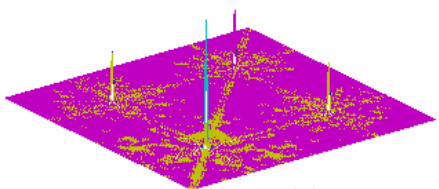

(e)

Fig. 4. TPSFs in the wavelet domain for full-sampling (a), chaotic undersampling (b) and random undersampling (c) with the compression ratio $r=0.05$ (b), chaotic undersampling (d) and random undersampling (e) with $r=0.1$

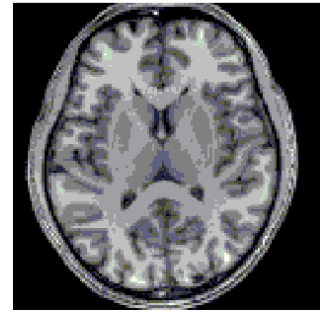

(a)

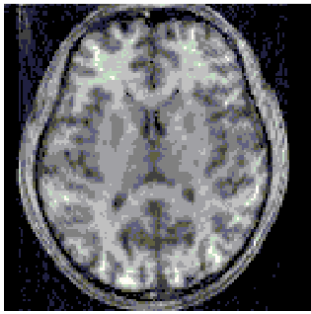

(b)

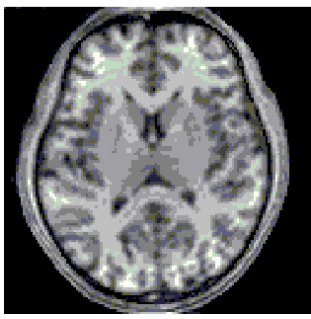

(d)

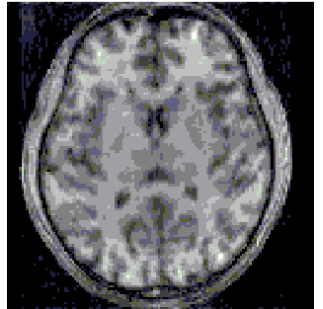

(c)

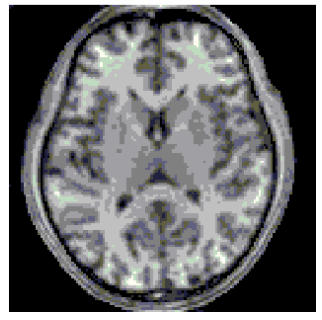

(e)

Fig. 5. Reconstructed images for different compression ratios: full-sampling (a), chaotic undersampling (b) and random undersampling (c) with $r=0.17$ (b), chaotic undersampling (d) and random undersampling (e) with $r=0.2$ 


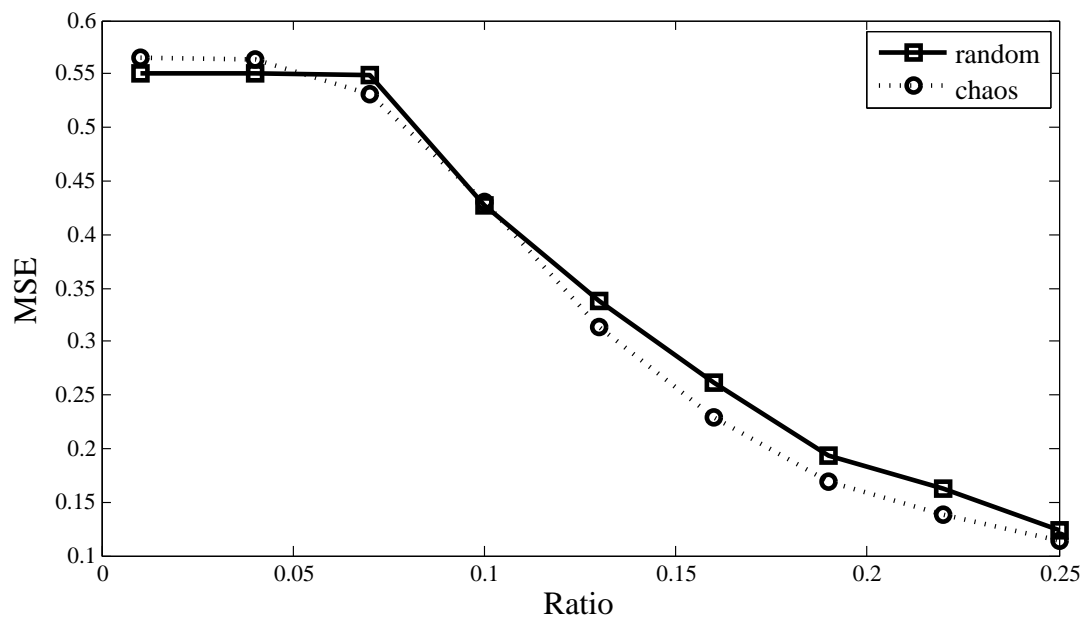

Fig. 6. Performance comparison between chaotic and random approach.

\section{REFERENCES}

[1] K. Pruessmann, M. Weiger, M. Scheidegger, and Boesiger, "SENSE: Sensitivity encoding for fast MRI," Magnetic Resonance in Medicine, vol. 42, pp. 952 - 962, 1999.

[2] W. E. Kyriakos, L. P. Panych, D. F. Kacher, C. F. Westin, S. M. Bao, R. V. Mulkern, and F. A. Jolesz, "Sensitivity profiles from an array of coils for encoding and reconstruction in parallel (SPACE RIP)," Magnetic Resonance in Medicine, vol. 44, no. 2, pp. 301-308, Aug 2000.

[3] M. Griswold, P. Jakob, M. Nittka, J. W. Goldfarb, and A. Haase, "Partially parallel imaging with localized sensitivities (PILS)," Magnetic Resonance in Medicine, vol. 44, pp. 602 - 609, 2000.

[4] L. Landini, V. Positano, and M. F. Santaelli, Advanced image processing in magnetic resonance imaging. Taylor \& Francis, 2005.

[5] D. Won and W. J. Manning, "Simutaneous acquisition of spatial harmonics (SMASH): fast imaging with radio frequency coil arrays," Magnetic Resonance in Medicine, vol. 38, pp. 591 - 603, October, 1997.

[6] M. A. Griswold, P. M. Jakob, R. M. Heidemann, M. Nittka, V. Jellus, J. Wang, B. Kiefer, and A. Haase, "Generalized autocalibrating partially parallel acquisitions (GRAPPA)," Magnetic Resonance in Medicine, vol. 47, no. 6, pp. 1202-1210, Jun 2002. [Online]. Available: http://dx.doi.org/10.1002/mrm.10171

[7] J. Tsao, P. Boesiger, and K. P. Pruessmann, " $k-t$ BLAST and $k$ - $t$ SENSE: Dynamic MRI with high frame rate exploiting spatiotemporal correlations," Magnetic Resonance in Medicine, vol. 50, no. 5, pp. 1031-1042, Nov 2003. [Online]. Available: http://dx.doi.org/10.1002/mrm.10611

[8] H. Jung, K. Sung, K. S. Nayak, E. Y. Kim, and J. C. Ye, "k-t FOCUSS: A general compressed sensing framework for high resolution dynamic MRI," Magnetic Resonance in Medicine, vol. 61, pp. 103 - 116, 2009.

[9] B. Madore, "UNFOLD-SENSE: A parallel MRI method with self-calibration and artifact suppression," Magnetic Resonance in Medicine, vol. 52, no. 2, pp. 310-320, Aug 2004. [Online]. Available: http://dx.doi.org/10.1002/mrm.20133

[10] E. Candes, J. Romberg, and T. Tao, "Robust uncertainty principles: Exact signal reconstruction from highly incomplete frequency information," IEEE Transactions on Information Theory, vol. 52, pp. 489509, 2006.

[11] D. Donoho, "Compressed sensing," IEEE Transactions on Information Theory, vol. 52, pp. 1289-1306, 2006.

[12] J. Tropp and A. Gilbert, "Signal recovery from random measurements via orthogonal matching pursuit," IEEE Transactions on Information Theory, vol. 53, pp. 4655-4666, 2007.

[13] M. Lustig, D. Donoho, and J. M. Pauly, "Sparse MRI: The application of compressed sensing for rapid MR imaging," Magnetic Resonance in Medicine, vol. 58, pp. 1182-1195, 2007.

[14] M. Lustig, J. M. Santos, D. L. Donoho, and J. M. Pauly, “ $k-t$ SPARSE:
High frame rate dynamic MRI exploiting spatio-temporal sparsity," in Proceedings of the ISMRM'06, 2006.

[15] A. Bilgin, Y. Kim, H. G. Lalgudi, T. P. Trouard, and M. I. Altbach, "Parallel magnetic resonance imaging using compressed sensing," in Proceedings of the SPIE on Applications of Digital Image Processing XXXI, A. G. Tescher, Ed., vol. 7073, 2008.

[16] D. V. Phong, N. Linh-Trung, H. V. Le, and M. N. Do, "Fast image acquisition in magnetic resonance imaging by chaotic compressed sensing," in 32nd Annual International IEEE EMBS Conference of the IEEE Engineering in Medicine and Biology Society (EMBC 2010), 2010, (submitted).

[17] R. A. DeVore, "Deterministic constructions of compressed sensing matrices," Journal of Complexity, vol. 23, pp. 918-925, 2007.

[18] V. Saligrama, "Deterministic designs with deterministic guarantees: Toeplitz compressed sensing matrices, sequence design and system identification," 2008, (Arxiv preprint- arXiv:0806.4958).

[19] L. Applebaum, S. Howard, S. Searle, and R. Calderbank, "Chirp sensing codes: Deterministic compressed sensing measurements for fast recovery," Applied and Computational Harmonic Analysis, vol. 26, pp. 283-290, 2009.

[20] N. Linh-Trung, D. V. Phong, Z. Hussain, H. Huynh, V. Morgan, and J. Gore, "Compressed sensing using chaos filters," in Australian Telecommunication Networks and Applications Conference (ATNAC 2008), 2008, pp. 219-223.

[21] E. Candes and J. Romberg, "Sparsity and incoherence in compressive sampling," Inverse Problems, vol. 23, pp. 969-985, 2007.

[22] "SENSE: sensitivity-encoding MRI Matlab tools." [Online]. Available: http://www.nmr.mgh.harvard.edu/f̃hlin/tool_sense.htm 